\title{
BPS bound states of D0-D6 and D0-D8 systems in a $B$-field
}

\author{
Edward Witten* \\ Department of Physics, Cal Tech, Pasadena, CA, USA, and \\ CIT-USC Center For Theoretical Physics \\ USC, Los Angeles CA, USA \\ E-mail: witten@ias.edu
}

ABStRaCT: The D0-D6 system, which is not supersymmetric in the absence of a NeveuSchwarz $B$-field, becomes supersymmetric if a suitable constant $B$-field is turned on. On one side of the supersymmetric locus, this system has a BPS bound state, and on the other side it does not. After compactification on $\mathbb{T}^{6}$, this gives a simple example in which the number of $1 / 8$ BPS states jumps as the moduli of the compactification are changed. The D0-D8 system in a $B$-field has two different supersymmetric loci, only one of which is continuously connected to the familiar supersymmetric D0-D8 system without a $B$-field. In a certain range, the D0-D8 system also has a BPS bound state. In the limit in which the $B$-field goes to infinity, supersymmetric D0-D6 and D0-D8 systems and their bound states can be studied using non-commutative Yang-Mills theory.

KEywords: Superstrings and Heterotic Strings, D-branes, Non-Commutative Geometry

${ }^{*}$ On leave from School of Natural Sciences, Institute for Advanced Study, Princeton, NJ 08540, USA. 


\section{Contents}

1. Introduction 1

2. Supersymmetric D0-D6 and D0-D8 systems 2

3. BPS states 5

3.1 Analysis of D0-D6 spectrum

3.2 Bound state for $\sum_{i} v_{i}>1 / 2$

3.3 Generalization for many branes

3.4 Analog for D0-D8 10

4. Comparison to non-commutative Yang-Mills theory 11

\section{Introduction}

D0-D $p$-brane systems in type-IIA superstrings (and more general $\mathrm{D} q-\mathrm{D} p$ systems) have been much studied. Some aspects have been reviewed in [1]. In the absence of a $B$-field, the D0-D0, D0-D4, and D0-D8 systems are supersymmetric, while the others are not. In the presence of a constant $B$-field, the condition for supersymmetry is modified. For example, the D0-D4 system in a constant $B$-field is supersymmetric precisely if the $B$-field is anti-self-dual [2]. More general conditions for supersymmetry in the presence of a $B$-field have been discussed in 3 , 国.

The D0-D2 system remains non supersymmetric in the presence of a $B$-field. However, as we will review in section 2, the D0-D6 system becomes supersymmetric when a suitable $B$-field is turned on. In the space of constant $B$-fields, there is a codimension one locus on which there is unbroken supersymmetry. In section 3 , we show that on one side of the supersymmetric locus, the D0-D6 system has a supersymmetric or BPS bound state, invariant under $1 / 8$ of the total supersymmetry; on the other side it does not. This result still holds if the D0-D6 system is compactified on $\mathbb{T}^{6}$; it demonstrates that in type-IIA superstring theory compactified on $\mathbb{T}^{6}$, the number of $1 / 8$ BPS states can jump as the moduli of the vacuum are changed. This jumping is analogous to the jumping of $1 / 4$ BPS states in $\mathcal{N}=4$ super Yang-Mills theory in four dimensions [5].

The D0-D8 system, on the other hand, is supersymmetric in the absence of a $B$ field. Turning on the $B$-field can preserve supersymmetry, and in addition there is a second supersymmetric locus of the D0-D8 system, not continuously connected to the theory with vanishing $B$-field. In a certain range, there is a supersymmetric D0-D8 bound state. 
It is possible to take the $B$-field to infinity and obtain supersymmetric D0-D6 and D0-D8 systems that can be described in non-commutative Yang-Mills theory, as in [2, 6. The relevant solutions of non-commutative Yang-Mills theory can be obtained by straightforwardly imitating arguments that have been given in the recent literature for the D1-D3, D0-D2, and D0-D4 cases [7]-[13]. Presumably, in a suitable range of $B$-fields where a BPS bound state exists, the non-commutative Yang-Mills theory could be used to demonstrate the decay of the D0-D6 and D0-D8 systems to a stable bound state, similarly to discussions of D0-D2 in 11, 13.

The analysis in section 4 is largely anticipated in 4 as I learned after submitting the original version of this paper to hep-th. In addition, the problem is $T$-dual to a problem treated in [14] involving D3-branes intersecting at angles [15.

\section{Supersymmetric D0-D6 and D0-D8 systems}

In type-IIA superstring theory, the supercharges that originate from left- and right-movers on the string worldsheet transform as spinors $Q_{\alpha}$ and $\widetilde{Q}^{\beta}$, respectively, of opposite chirality. For a linear combination $\sum_{\alpha} \epsilon^{\alpha} Q_{\alpha}+\sum_{\beta} \widetilde{\epsilon}_{\beta} \widetilde{Q}^{\beta}$ to be unbroken in the presence of a D-brane, a certain condition must be obeyed, depending on the brane.

For example, in the field of a D0-brane at rest, with $x^{0}$ being the time direction and $x^{1}, \ldots, x^{9}$ the space directions, the condition is

$$
\widetilde{\epsilon}_{\beta}=\Gamma_{\beta \alpha}^{0} \epsilon^{\alpha},
$$

where $\Gamma^{i}$ are Dirac gamma matrices. This equation has a simple (and well known; see for instance [1]) interpretation. Consider an open string that ends on the D0-brane. Because the worldsheet field $X^{0}$ corresponding to $x^{0}$ obeys Dirichlet boundary conditions and the other fields $X^{i}, i>0$, obey Neumann boundary conditions, the worldsheet modes when reflected from the end of the string are multiplied by a matrix that (in the vector representation of the ten-dimensional Lorentz group) is $\operatorname{diag}(1,-1,-1, \ldots,-1)$. In the spinor representation, this matrix is $\Gamma^{0}$.

Now consider a D6-brane whose world-volume fills the directions $x^{0}, x^{1}, \ldots, x^{6}$, but with vanishing $B$-field. The condition for unbroken supersymmetry in the field of such a brane is

$$
\tilde{\epsilon}_{\beta}=\left(\Gamma^{0} \Gamma^{1} \cdots \Gamma^{6}\right)_{\beta \alpha} \epsilon^{\alpha} .
$$

This reflects the fact that for a string ending on the D6-brane, $X^{0}, \ldots, X^{6}$ obey Dirichlet boundary conditions and the others obey Neumann boundary conditions, so the reflection matrix is $\operatorname{diag}(-1,-1, \ldots,-1,1,1,1)$, with -1 's for $x^{0}, \cdots, x^{6}$.

In the presence of both a D0-brane and a D6-brane, the unbroken supersymmetries must obey both conditions. Combining them, we get

$$
\left(\Gamma^{1} \Gamma^{2} \cdots \Gamma^{6}\right) \epsilon=\epsilon .
$$

This equation has no non-vanishing solutions, since $N=\Gamma^{1} \Gamma^{2} \cdots \Gamma^{6}$ obeys $N^{2}=-1$ and has all eigenvalues $\pm i$. This shows that, without the $B$-field, the D0-D6 system is not supersymmetric. 
As the above explanation makes clear, the matrix $N$ that appears here has a simple interpretation: it represents the action on spinors of the Lorentz transformation that acts as -1 on $x^{1}, \ldots, x^{6}$ and +1 on the other coordinates. We can think of this group element as an element of the center of the rotation group $\mathrm{SO}(6)$ that acts on $x^{1}, \ldots, x^{6}$.

Now, let us turn on a constant $B$-field in the directions $x^{1}, \ldots, x^{6}$. Consider a string that ends on the D6-brane in the presence of the $B$-field. In the RNS description, the worldsheet bosons and fermions $x^{i}, \psi^{i}, i=1, \ldots, 6$, when they are reflected from the string end, undergo a rotation by an element $M$ of $\mathrm{SO}(6)$ that depends on $B$. We can pick a coordinate system in which $B$ is the sum of three $2 \times 2$ blocks, with the $i^{\text {th }}$ such block taking the form

$$
\frac{1}{2 \pi \alpha^{\prime}}\left(\begin{array}{cc}
0 & -b_{i} \\
b_{i} & 0
\end{array}\right)
$$

Then [16, 17] $M$ is likewise the sum of three $2 \times 2$ blocks, with the $i^{\text {th }}$ block being

$$
\left(\begin{array}{cc}
\cos 2 \pi v_{i} & \sin 2 \pi v_{i} \\
-\sin 2 \pi v_{i} & \cos 2 \pi v_{i}
\end{array}\right)
$$

with

$$
e^{2 \pi i v_{i}}=\frac{1+i b_{i}}{1-i b_{i}}, \quad-\frac{1}{2}<v_{i}<\frac{1}{2} .
$$

$\left(v_{i}\right.$ has here been shifted by $1 / 2$ relative to [2].)

When we allow for this extra rotation matrix, the worldsheet modes of a string ending on a D6-brane are rotated not just by the Lorentz transformation that in the spinor representation is $\Gamma^{0} \Gamma^{1} \cdots \Gamma^{6}$; there is an extra factor $\rho(M)$, where $\rho(M)$ is simply $M$ written in the spinor representation. (2.2) becomes

$$
\widetilde{\epsilon}_{\beta}=\left(\Gamma^{0} \Gamma^{1} \cdots \Gamma^{6} \rho(M)\right)_{\beta \alpha} \epsilon^{\alpha},
$$

and (2.3) becomes

$$
\left(\Gamma^{1} \Gamma^{2} \cdots \Gamma^{6} \rho(M)\right) \epsilon=\epsilon,
$$

or more simply

$$
\rho(-M) \epsilon=\epsilon,
$$

since $\Gamma^{1} \Gamma^{2} \cdots \Gamma^{6}=\rho(-1)$ represents -1 in the spinor representation.

Thus, the condition for unbroken supersymmetry is that the $\mathrm{SO}(6)$ element $-M$ must, in the spinor representation, have +1 as one of its eigenvalues. The condition for this is familiar: $-M$ must be an element of some $\mathrm{SU}(3)$ subgroup of $\mathrm{SO}(6)$. If we complexify the space generated by $x^{1}, \ldots, x^{6}$ to make a copy of $\mathbb{C}^{6}$, then the $\mathrm{SU}(3)$ will leave fixed a three-dimensional subspace $\mathbb{C}^{3} \subset \mathbb{C}^{6}$. This $\mathbb{C}^{3}$ must be generated by eigenspaces of the matrix $M$. If the three blocks in (2.4) are assumed to act on the 1-2, 3-4, and 5-6 planes, then the eigenspaces of $M$ are generated by $x^{1} \pm i x^{2}, x^{3} \pm i x^{4}$, and $x^{5} \pm i x^{6}$. The relevant $\mathbb{C}^{3}$ is generated by $x^{1} \pm i x^{2}, x^{3} \pm i x^{4}$, and $x^{5} \pm i x^{6}$ with some specific choices of the signs. This $\mathbb{C}^{3}$ is invariant under a $\mathrm{U}(3)$ that contains $-M$; the condition that $-M$ is actually in $\mathrm{SU}(3)$ is that, if it is understand as a $3 \times 3$ diagonal matrix with eigenvalues $-e^{ \pm 2 \pi i v_{k}}$, 
$k=1,2,3$ (with some choices of sign), its determinant must be 1 . The condition in other words is that

$$
\pm v_{1} \pm v_{2} \pm v_{3}= \pm \frac{1}{2}
$$

with some choices of the signs. In fact, by possibly reversing orientation in the 1-2, 3-4, and 5-6 planes, we can always arrange so that the signs are all positive, and the condition for unbroken supersymmetry is

$$
v_{1}+v_{2}+v_{3}=\frac{1}{2}
$$

When this condition is obeyed and the $v_{i}$ are otherwise generic, (2.9) is obeyed for onefourth of the components of $\epsilon$. Allowing also for (2.7), one-eighth of the 32 supersymmetries are unbroken. Thus, there are four unbroken supersymmetries in all.

Comparison To D0-D4. Let us now compare this to the familiar result for D0-D4. For D0-D4, the matrix $-M$ has eigenvalues $-e^{ \pm 2 \pi i v_{k}}, k=1,2$. The condition for its determinant to be 1 as a $\mathrm{U}(2)$ matrix is

$$
v_{1} \pm v_{2}=0
$$

With the basis $x^{1}, \ldots, x^{4}$ oriented in the fashion assumed in [2, the sign is + . (2.12) together with (2.5) means that $B_{1}=-B_{2}$ so that the $B$-field is anti-self-dual.

(2.12) implies that the eigenvalues of $-M$ are not distinct, so when (2.12) holds, $-M$ commutes with a subgroup of $\mathrm{SO}(4)$ of larger than the generic dimension and is on a conjugacy class in $\mathrm{SO}(4)$ smaller than the generic dimension. Indeed, when (2.12) is obeyed, $-M$ takes values in one $\mathrm{SU}(2)$ factor of $\mathrm{SO}(4)=\mathrm{SU}(2)_{L} \times \mathrm{SU}(2)_{R}$. The subgroup of $\mathrm{SO}(4)$ that commutes with $-M$ is then not $\mathrm{U}(1) \times \mathrm{U}(1)$ (as it is for generic $M$ ) but $\mathrm{SU}(2) \times \mathrm{U}(1)$. As the dimension of the commuting subgroup of $-M$ increases by two when (2.12) holds, the dimension of its orbit in $\mathrm{SO}(4)$ drops by two. So while (2.12) puts a single real condition on the $v_{i}$, the space of $M$ 's for which it holds is of real codimension three. In terms of $B$, this statement is easy to explain: for $B$ to be anti-self-dual is three real conditions.

For the D0-D6 system, the situation is different. A generic $-M$ commutes with $\mathrm{U}(1) \times$ $\mathrm{U}(1) \times \mathrm{U}(1) \subset \mathrm{SO}(6)$. When $(2.10)$ is obeyed, the eigenvalues of $-M$ remain generically distinct and the stabilizer of $-M$ is still generically $\mathrm{U}(1) \times \mathrm{U}(1) \times \mathrm{U}(1)$. The dimension of the orbit does not jump, and so (2.10) places one real condition on $M$ just as it does on the $v_{i}$. The D0-D6 system is supersymmetric on a locus of real codimension one in the space of $B$-fields. This is a crucial fact, for it will make it possible later for the number of BPS states to jump in crossing this locus.

Analog For D0-D2 and D0-D8. For the D0-D2 system, there is only a single rotation parameter $v$. The eigenvalues of $-M$ are $-e^{ \pm 2 \pi i v}$, and the determinant of $-M$ as an element of $\mathrm{U}(1)$ is $-e^{ \pm 2 \pi i v}$ (with the sign depending on which $\mathrm{U}(1)$ we pick). As $|v|<1 / 2$, this cannot equal 1 , so turning on a $B$-field does not restore supersymmetry for the D0D2 system. However, in the limit of $|B| \rightarrow \infty$, one has $|v| \rightarrow 1 / 2$, and supersymmetry is restored. 
For the D0-D8 system, there are four rotation parameters $v_{i}$. The eigenvalues of $-M$ are $-e^{ \pm 2 \pi i v_{i}}$. The condition for $-M$ to have determinant 1 in $\mathrm{U}(4)$ is that, with some choice of the signs,

$$
\pm v_{1} \pm v_{2} \pm v_{3} \pm v_{4}=\text { integer }
$$

As $\left|v_{i}\right|<1 / 2$, there are two essentially different cases. There is a supersymmetric locus with

$$
\pm v_{1} \pm v_{2} \pm v_{3} \pm v_{4}=0
$$

This includes as a special case the possibility that $B_{i}=v_{i}=0$ for all $i$, which is the familiar supersymmetric D0-D8 system without a $B$-field. The more novel possibility is

$$
\pm v_{1} \pm v_{2} \pm v_{3} \pm v_{4}= \pm 1
$$

Both the conventional supersymmetric locus and the novel one are of real codimension 1 in the space of $B$-fields, since neither (2.14) nor (2.15) forces any degeneracies in the eigenvalues of $-M$. Once again, we can make all of the signs positive with a suitable choice of orientations. When (2.14) or (2.15) is satisfied and the $v_{i}$ are otherwise generic, there are two unbroken supersymmetries.

\section{BPS states}

\subsection{Analysis of D0-D6 spectrum}

We will now analyze the effective low energy physics of the supersymmetric D0-D6 system. We will show that this system supports a massless chiral supermultiplet. We will also show that the ground state energy of the D0-D6 system in the Neveu-Schwarz sector changes sign as one crosses the supersymmetric locus. (This was seen in [3].) We will then argue, based on this, that on one side of the supersymmetric locus there is a supersymmetric or BPS bound state, and on the other side there is not.

In the Ramond sector, we can analyze the situation as follows. We work in light cone gauge with transverse oscillators $X^{1}, \ldots, X^{8}$ and world-sheet superpartners $\psi^{1}, \ldots, \psi^{8}$. The ground state energy vanishes in the Ramond sector because of worldsheet supersymmetry. In the notation of the last section, the worldsheet fermions $\psi^{7}$ and $\psi^{8}$ (superpartners of $X^{7}$ and $X^{8}$, which describe motion orthogonal to the D6-brane) have zero modes. The other worldsheet fermions obey twisted boundary conditions that depend on the $B$-field; for generic $v_{i}$, they have no zero modes. Quantization of the $\psi^{7}$ and $\psi^{8}$ zero modes give a pair of massless states whose spin (under rotations of the $7-8$ plane) is $\pm 1 / 2$. Precisely one of these two states survives the GSO projection.

Hence, for a supersymmetric configuration, there must also be a massless state in the Neveu-Schwarz sector, making part of a massless vector or chiral supermultiplet. Let us see how this comes about. First we consider the case that all $v_{i}$ are positive with

$$
v_{1}+v_{2}+v_{3}=\frac{1}{2}
$$


We recall that a complex boson $X$ with modes $X_{n+1 / 2+\theta},|\theta| \leq 1 / 2$, has a ground state energy

$$
\frac{1}{24}-\frac{\theta^{2}}{2}
$$

A complex fermion $\psi$ with modes $\psi_{n+1 / 2+\theta}$ likewise has a ground state energy

$$
-\frac{1}{24}+\frac{\theta^{2}}{2}
$$

In the problem at hand, for $X^{1}+i X^{2}, X^{3}+i X^{4}$, and $X^{5}+i X^{6}$, we have $\theta$ equal to $v_{1}$, $v_{2}$, and $v_{3}$, respectively. For $X^{7}+i X^{8}, \theta=1 / 2$. So the bosonic ground state energy is

$$
\sum_{i=1}^{3}\left(\frac{1}{24}-\frac{v_{i}^{2}}{2}\right)-\frac{1}{12} .
$$

For the fermions in the Neveu-Schwarz sector, the corresponding values of $\theta$ are $v_{i}-1 / 2$ (for $i=1,2,3$ ) and 0 . So the fermionic ground state energy is

$$
\sum_{i=1}^{3}\left(-\frac{1}{24}+\frac{\left(1 / 2-v_{i}\right)^{2}}{2}\right)-\frac{1}{24} .
$$

Adding these up, we find the ground state energy to be

$$
\frac{1}{4}-\frac{1}{2} \sum_{i=1}^{3} v_{i} .
$$

So in the supersymmetric case, with $\sum_{i} v_{i}=1 / 2$, the ground state energy vanishes for D0-D6 strings in the NS sector. There are no worldsheet zero modes to be quantized, so there is a unique massless D0-D6 state in this sector. It has vanishing spin since, for example, the filled fermi sea in this problem is invariant under a reflection in the 7-8 plane. Since we found a massless fermi state in the Ramond sector, this unique massless state in the NS sector must survive the GSO projection. When $\sum_{i} v_{i}=1 / 2$, the massless bosonic and fermionic states from the NS and R sectors combine with similar states of D6-D0 strings to make a massless chiral multiplet.

Before analyzing the implications of this, let us first consider a more general situation with unbroken supersymmetry. There is no loss of essential generality in picking positive signs in the supersymmetry condition as in (2.11), but we should not necessarily assume that all $v_{i}$ are positive. Since $\left|v_{i}\right|<1 / 2$, at least two $v_{i}$ must be positive if $v_{1}+v_{2}+v_{3}=1 / 2$. So the remaining essential case is that $1 / 2>v_{1}, v_{2} \geq 0$ and $0>v_{3}>-1 / 2$. These conditions imply

$$
\left|v_{3}\right|<v_{1}, v_{2} \text {. }
$$

With $v_{3}$ negative, one must replace $v_{3}$ by $1-v_{3}$ in (3.5), and the ground state energy comes out to be

$$
-\left|v_{3}\right|
$$


So the NS sector is tachyonic in this case. Spacetime supersymmetry ensures that this tachyon is removed by the GSO projection. The worldsheet fermions have modes $\psi_{n \pm v_{i}}$ (for $\psi^{1}, \ldots, \psi^{6}$ ) and $\psi_{n+1 / 2}\left(\right.$ for $\psi^{7}, \psi^{8}$ ). In view of (3.7), this means that the lowest energy fermion creation operator has energy precisely $\left|v_{3}\right|$. Acting with this operator on the tachyonic ground state, we get a unique GSO-even state of zero energy and zero spin. This completes the demonstration that in the supersymmetric case, quantization of the D0-D6 NS sector always gives a single massless scalar state.

\subsection{Bound state for $\sum_{i} v_{i}>1 / 2$}

Let us describe the low energy effective field theory of the D0-D6 system. We think of it as a field theory — really a quantum mechanical system — on the D0 world-line.

This theory has four unbroken supercharges - the supercharges left unbroken by the combined D0-D6 system for $\sum_{i} v_{i}=1 / 2$. It has a $\mathrm{U}(1)$ vector multiplet, which arises in quantizing the 0-0 strings. Apart from the gauge field, this vector multiplet contains fermions $\lambda$ and scalars $\vec{\sigma}$. (One can think of the scalars as arising by dimensional reduction of a four-dimenional vector multiplet to $0+1$ dimensions. For a discussion of the possible low energy couplings of a vector multiplet in $0+1$ dimensions, see [18].) The low energy theory also has a chiral multiplet $\Phi=\phi+\theta \psi+\cdots$ that arises, as we have just seen, by quantizing the 0-6 and 6-0 strings. Finally, there are three more massless chiral multiplets $T_{\alpha}, \alpha=1, \ldots, 3$ that arise from quantizing the 0-0 strings and describe the motion of the D0-brane in the directions $x^{1}, \ldots, x^{6}$ tangent to the D6-brane. These are Goldstone modes of an exact symmetry of spacetime translations, and hence decouple from the low energy dynamics.

The physics of this system clearly depends very much on the real parameter $r=$ $\sum_{i} v_{i}-1 / 2$. At $r=0$, supersymmetry is unbroken. For $r<0$, the D0-D6 system is stable (as the NS ground state energy is positive) but not supersymmetric. The supersymmetry breaking is small if $|r|$ is very small, and it must be possible to interpret it as spontaneous breaking of supersymmetry in the low energy effective theory. For $r>0$, the D0-D6 system is tachyonic and unstable. It must decay to something else with the same quantum numbers. For small $r$, it is reasonable to expect to be able to describe the stable state in the low energy effective theory.

With four supercharges, most coupling parameters are complex, and it is somewhat unusual to see a real parameter playing an important role. However, in this case, it is easy to see, by analogy with known behavior of the D0-D4 system [19, what effective field theory has the right properties: the parameter $r$ is the Fayet-Iliopoulos coupling of the low energy $\mathrm{U}(1)$ gauge theory. The potential energy function $V$ of the low energy field theory then has a term proportional to $D^{2}$, where $D=\bar{\phi} \phi-r$. Thus

$$
V \sim(\bar{\phi} \phi-r)^{2}
$$

Here we see that for $r<0$, the vacuum at $\phi=0$ is stable but has positive energy and so describes a system with spontaneously broken supersymmetry. In this state, the U(1) gauge symmetry is unbroken; we interpret this state as the D0-D6 ground state. For $r=0$, the $\mathrm{U}(1)$ is still unbroken, and supersymmetry is also unbroken. But for $r>0$, there is 
a stable, supersymmetric vacuum with $|\phi|=\sqrt{r}$; this vacuum has spontaneously broken $\mathrm{U}(1)$ gauge symmetry, and the phase of $\phi$ can be eliminated by a gauge transformation. This is the stable vacuum that arises from the D0-D6 system by tachyon condensation when $r$ is slightly greater than zero.

Apart from the Goldstone multiplets $T_{\alpha}$ (which decouple from the low energy dynamics), the state with $r>0$ and $|\phi|=\sqrt{r}$ is massive: all components of the vector and chiral multiplets have mass in expanding around this vacuum. Hence, though we found this state by examination of the classical potential, its existence is stable against quantum corrections. There is no candidate for what a Goldstone fermion might be in the event of supersymmetry breaking due to quantum corrections. (For $r<0$, the Goldstone fermion is part of the vector multiplet, but for $r>0$, the vector multiplet gets mass by a Higgs effect.)

The following question is puzzling at first sight. For $r>0$, the D0-D6 system, with the translations factored out, has a unique, massive, supersymmetric vacuum, so that the supersymmetric index is $\operatorname{Tr}(-1)^{F}=1$. For $r<0$, supersymmetry is spontaneously broken, so $\operatorname{Tr}(-1)^{F}=0$. How can $\operatorname{Tr}(-1)^{F}$ jump? This jumping depends on the following facts. For $r \leq 0$, the effective U(1) gauge theory also has a Coulomb branch, in which the scalar fields $\vec{\sigma}$ in the vector multiplet get a vacuum expectation value. This Coulomb branch, of course, describes the motion of the D0-brane away from the D6-brane. It is absent for $r>0$, because there is a term $\vec{\sigma}^{2}|\phi|^{2}$ in the classical potential; this term prevents $\vec{\sigma}$ from getting a vacuum expectation value when $\phi$ has one. (More informally, the supersymmetric ground state for $r>0$ is a D0-D6 bound state, so it does not admit any deformation to a Coulomb branch in which the two branes separate.) For $r \leq 0$, because of the $\vec{\sigma}^{2}|\phi|^{2}$ interaction, the field $\vec{\sigma}$ can only go to infinity at finite cost of energy if $\phi$ vanishes. At this point, the classical energy is proportional to $r^{2}$, because of (3.9). If $\vec{\sigma}$ can go to infinity, the theory has a continuous spectrum. Hence, the theory has a continuous spectrum above a threshold proportional to $r^{2}$. At $r=0$, this threshold begins at zero energy. This means that at $r=0$, the counting of zero energy states breaks down, and $\operatorname{Tr}(-1)^{F}$ can jump.

Though we have formulated our discussion for a D0-D6 system in $\mathbb{R}^{10}$, the discussion is not much modified by compactification as long as a sufficiently strong $B$-field can be turned on. For instance, we could consider compactification to $\mathbb{R}^{4} \times \mathbb{T}^{6}$ with the D6-brane wrapped on $\mathbb{T}^{6}$. Our discussion carries over to this case directly; the supersymmetric D0D6 bound state that arises for $r>0$ is a finite energy BPS state. It is a $1 / 8$ BPS state invariant that is under $1 / 8$ of the 32 supercharges of the toroidally compactified type-IIA superstring theory. This bound state is absent for $r<0$, showing explicitly that in string compactification to four dimensions with 32 unbroken supersymmetries, the number of $1 / 8$ BPS states can jump as the parameters of the vacuum are modified. Such jumping, which might be important in some aspects of the counting of microscopic states of BPS black holes in four dimensions, is analogous to the jumping of $1 / 4$ BPS states in $\mathcal{N}=4$ super Yang-Mills theory in four dimensions [0].

\subsection{Generalization for many branes}

We can readily generalize these results to the case of $N$ D0-branes interacting with $M$ coincident D6-branes. 
The gauge group of the D0-branes is now $\mathrm{U}(N)$. The D6-branes support a $\mathrm{U}(M)$ gauge symmetry, which appears as a global symmetry in the D0-brane quantum mechanics. The quantization of the 0-6 strings is the same as before, except that we must include ChanPaton factors. The upshot is that, on the supersymmetric locus, the massless 0-6 and 6-0 strings are a multiplet of massless chiral superfields $\Phi^{i}{ }_{k}, i=1, \ldots, N, k=1, \ldots, M$ transforming as $(N, \bar{M})$ of $\mathrm{U}(N) \times \mathrm{U}(M)$.

Allowing for the $\mathrm{U}(N)$ Chan-Paton factors, the massless 0-0 strings are, apart from the $\mathrm{U}(N)$ vector multiplet, a trio of superfields $T_{\alpha}$ transforming in the adjoint representation of $\mathrm{U}(N)$. In contrast to the $\mathrm{U}(1)$ case, where the $T_{\alpha}$ are neutral and decouple, for $\mathrm{U}(N)$ they do not decouple. The $T_{\alpha}$ have a superpotential $W=\operatorname{Tr} T_{1}\left[T_{2}, T_{3}\right]$, which follows from dimensional reduction from ten-dimensional super-Yang-Mills theory to $0+1$ dimensions.

If we write $\Phi^{i}{ }_{k}=\phi_{k}^{i}+\cdots$ where the $\phi^{i}{ }_{k}$ are the $\theta=0$ components of the superfields, and likewise $T_{\alpha}=t_{\alpha}+\cdots$, then the $D$-fields are

$$
D_{j}^{i}=\sum_{k} \phi_{k}^{i} \bar{\phi}^{k}{ }_{j}+\sum_{\alpha}\left[t_{\alpha}, \bar{t}_{\alpha}\right]_{j}^{i}-r \delta^{i}{ }_{j}
$$

(Here $\bar{\phi}^{k}{ }_{j}, k=1, \ldots, M, j=1, \ldots, N$ transforms of course as $(\bar{N}, M)$.) A classical supersymmetric state is a solution of $D^{i}{ }_{j}=0$ together with $\left[t_{\alpha}, t_{\beta}\right]=0$ (the last condition corresponds to $\left.\partial W / \partial T_{\alpha}=0\right)$. Since the trace of the $\phi \bar{\phi}$ term in $D$ is positive definite, while the $[t, \bar{t}]$ term is traceless, such states exist if and only if $r \geq 0$. Moreover, if $r=0$, by taking the trace of the equation $D^{i}{ }_{j}=0$ we learn that $\phi^{i}{ }_{k}=0$, so that the 0-6 strings play no role and the discussion collapses to an analysis of the 0-0 strings and their zero modes $t_{\alpha} \cdot{ }^{1}$ So the interesting case is $r>0$. The moduli space $\mathcal{M}$ of classical supersymmetric ground states is the space of supersymmetric classical states, divided by $\mathrm{U}(N)$. (Quantum supersymmetric ground states must be found by studying a suitable quantum mechanics on $\mathcal{M}$. We will not investigate this here.)

This description of the space of classical D0-D6 bound states is somewhat analogous to the ADHM description of the moduli space of classical D0-D4 bound states, that is, instantons, and the generalization of the ADHM construction to instantons on non-commutative $\mathbb{R}^{4}$, that is instantons with a Fayet-Iliopoulos term [20]. One difference is that D0-D4 bound states are interesting both in the absence of a Fayet-Iliopoulos term (classical instantons) and in the presence of one (instantons on non-commutative space), while for D0-D6, bound states exist only in the presence of a Fayet-Iliopoulos term. Moreover, of course, for D0-D4, there are bound states for all values of $B$ (or all values for which $B$ is not anti-self-dual if one has only one D4-brane), while for D0-D6, the $B$-field must obey a certain inequality. Finally, by taking the trace of the equation $D^{i}{ }_{j}=0$, we learn that even for $r>0$, the $\phi_{k}^{i}$ are bounded in absolute value, which corresponds roughly to an upper bound on the "size" of a bound state; there is of course no such bound for instantons.

\footnotetext{
${ }^{1}$ If the $\phi^{i}{ }_{k}$ vanish and $\left[t_{\alpha}, t_{\beta}\right]=0$, then the D0-branes are free to separate from the D6-branes, by giving an expectation value to scalars in the $\mathrm{U}(N)$ vector multiplet. Such configurations do not describe D0-D6 bound states.
} 
It is perhaps of some interest for $r>0$ to consider the case that the $T_{\alpha}$ are all zero, corresponding roughly to all D0-branes being at the same point in space. Such solutions exist if and only if $M \geq N$. For this case, the equation $D=0$ says that the $M$-component row vectors whose components are $\phi_{k}^{i} / \sqrt{r}$ for fixed $i$ (the $M$ components of the vector being labeled by $k=1, \ldots, M)$ are orthonormal. The space of such $N$-plets of orthonormal vectors, modulo the action of $\mathrm{U}(N)$, is the Grassmannian $\mathrm{U}(M) / \mathrm{U}(N) \times \mathrm{U}(M-N)$ of complex $N$-planes in $\mathbb{C}^{M}$. So this is the moduli space of supersymmetric states with $T_{\alpha}=0$.

\subsection{Analog for D0-D8}

Now let us consider the analogous issues for the D0-D8 system. We recall that there are two distinct supersymmetric loci of the D0-D8 system, governed, respectively, by (2.14) and (2.15). The first one contains as a special case the standard supersymmetric D0-D8 system with $B=0$. In this case, the ground state energy in the NS sector is strictly positive, and there are no massless bosons. We therefore concentrate on the second supersymmetric locus. Without essential loss of generality, we can pick orientations so that the signs in (2.15) are all positive. We moreover will consider only the case that the $v_{i}$ are all positive, and leave the interested reader to examine more general cases, by analogy with the last paragraph of section 3.1. Thus we assume

$$
0 \leq v_{i}<\frac{1}{2}, \quad \sum_{i=1}^{4} v_{i}=1 .
$$

The analog of (3.4) for the bosonic contribution to the NS ground state energy of the D0-D8 system is

$$
\sum_{i=1}^{4}\left(\frac{1}{24}-\frac{v_{i}^{2}}{2}\right)
$$

while the analog of (3.5) for the fermionic contribution is

$$
\sum_{i=1}^{4}\left(-\frac{1}{24}+\frac{\left(1 / 2-v_{i}\right)^{2}}{2}\right) .
$$

Adding these, the total ground state energy is

$$
\frac{1}{2}\left(1-\sum_{i} v_{i}\right)
$$

Thus, we see the expected vanishing of the ground state energy when $\sum_{i} v_{i}=1$. Moreover, for $\sum_{i} v_{i}<1$, the D0-D8 system is stable, though not supersymmetric, and there is not evidence of a BPS bound state. For $\sum_{i} v_{i}>1$, the D0-D 8 system is unstable and presumably decays to a supersymmetric or BPS ground state. The effective low energy theory is less constrained by supersymmetry, as there are only two supercharges. 


\section{Comparison to non-commutative Yang-Mills theory}

The conditions for unbroken supersymmetry found in section 2 do not require the $B$-field to be large in string units; it suffices for it to be of order 1 . However, while preserving unbroken supersymmetry, it is possible to take $B \rightarrow \infty$ and thus to make contact with non-commutative Yang-Mills theory.

In the limit that $b_{i} \rightarrow \pm \infty$, the rotation angles $v_{i}$ defined in (2.6) behave as

$$
v_{i} \longrightarrow \frac{1}{2} \operatorname{sign} b_{i}-\frac{1}{\pi b_{i}} \text {. }
$$

In this limit, the supersymmetry condition (2.10) of the D0-D6 system becomes simply

$$
\pm \frac{1}{b_{1}} \pm \frac{1}{b_{2}} \pm \frac{1}{b_{3}}=0 \text {. }
$$

Likewise, the supersymmetry condition (2.14) of the D0-D8 system reduces to

$$
\pm \frac{1}{b_{1}} \pm \frac{1}{b_{2}} \pm \frac{1}{b_{3}} \pm \frac{1}{b_{4}}=0 \text {. }
$$

We want to interpret these conditions in terms of non-commutative Yang-Mills theory.

To do so, we should consider a suitable solution of non-commutative Yang-Mills theory describing the D0-D6 system, find the condition for this solution to be supersymmetric, and compare this condition to (4.1). (The D0-D8 system, and its comparison to (4.3), can be treated in precisely the same way.) Which solution we want is quite clear from several recent papers [7] - [13]. To explain the situation starting from matrix theory (as in [21]-22]), we describe the D6-brane with a $B$-field via matrices $X^{i}, i=1, \ldots, 6$ obeying $\left[X^{i}, X^{j}\right]=i \theta^{i j}$. This description is familiar in matrix theory [30, 31, 32]. We likewise describe the D0-brane by $1 \times 1$ matrices with $X^{i}$ being multiples of the identity. The D0D6 brane solution is described in matrix theory by taking the direct sum of the two sets of matrices. It can be reinterpreted as a solution of rank one non-commutative Yang-Mills theory with

$$
\hat{F}_{i j}=-\Pi \theta_{i j}^{-1},
$$

with $\Pi$ the projector onto a single state in Hilbert space (this state originates with the $1 \times 1$ matrices used to describe the D0-brane). For details, the reader may consult [11, 12, 13].

Next let us find the condition for this solution to be supersymmetric. In general, the transformation law for the gluino field $\lambda$ in non-commutative super-Yang-Mills theory is $\delta \lambda=\hat{\Gamma}^{i j} \hat{F}_{i j} \epsilon+\epsilon^{\prime}$, where $\epsilon$ and $\epsilon^{\prime}$ are, respectively, the unbroken and spontaneously broken supersymmetries. Here $\hat{\Gamma}^{i j}=\frac{1}{2}\left[\hat{\Gamma}^{i}, \hat{\Gamma}^{j}\right]$, where

$$
\left\{\hat{\Gamma}^{i}, \hat{\Gamma}^{j}\right\}=2 G^{i j},
$$

with $G^{i j}$ being the "open string metric" used in the non-commutative super Yang-Mills theory. The condition for unbroken supersymmetry is

$$
0=\hat{F}_{i j} \hat{\Gamma}^{i j} \epsilon+\epsilon^{\prime} .
$$


For the solution we are considering, $\hat{F}$ vanishes at infinity, and hence we can assume that $\epsilon^{\prime}=0$. Given (4.4), the condition for unbroken supersymmetry thus reduces to

$$
\hat{\Gamma}^{i j} \theta_{i j}^{-1} \epsilon=0
$$

To compare with the result of section 2, we must express this condition in terms of the closed string variables - the metric $g_{i j}$ and $B$-field $B_{i j}$. In the following discussion, we will work entirely in the six-dimensional spatial volume of the D6-brane (at fixed time) with coordinates $x^{1}, \ldots, x^{6}$. We assume that $B$ is invertible in this six-dimensional space; in fact, we assume that the "eigenvalues" $b_{1}, b_{2}$, and $b_{3}$ are all tending to $\pm \infty$. In the $\alpha^{\prime} \rightarrow 0$ limit, the noncommutativity parameter $\theta$ and open string metric $G$ are [2]

$$
\theta=B^{-1}, \quad G_{i j}=-\left(2 \pi \alpha^{\prime}\right)^{2}\left(B g^{-1} B\right)_{i j} .
$$

We also introduce gamma matrices $\Gamma^{i}$ appropriate to the closed string metric:

$$
\left\{\Gamma^{i}, \Gamma^{j}\right\}=2 g^{i j}, \Gamma_{i}=g_{i k} \Gamma^{k}, \quad \Gamma_{i j}=\frac{1}{2}\left[\Gamma_{i}, \Gamma_{j}\right]
$$

Given the relation between $G$ and $g$, we can take the $\hat{\Gamma}^{i}$ to be

$$
\hat{\Gamma}^{i}=\left(2 \pi \alpha^{\prime}\right)^{-1} \theta^{i j} g_{j k} \Gamma^{k}
$$

The condition (4.7) of unbroken supersymmetry can hence be expressed in terms of closed string variables:

$$
\Gamma_{i j}\left(B^{-1}\right)^{i j} \epsilon=0
$$

If $B$ is the direct sum of $2 \times 2$ blocks with "eigenvalues" $b_{1}, b_{2}, b_{3}$, then $B^{-1}$ is likewise a direct sum of $2 \times 2$ blocks with eigenvalues $-b_{i}^{-1}, i=1,2,3$. So (4.11) becomes

$$
\left(b_{1}^{-1} \Gamma_{12}+b_{2}^{-1} \Gamma_{34}+b_{3}^{-1} \Gamma_{56}\right) \epsilon=0 .
$$

The matrices $\Gamma_{12}, \Gamma_{34}$, and $\Gamma_{56}$ commute and have eigenvalues $\pm i$, with each set of signs $( \pm i, \pm i, \pm i)$ arising for some eigenvector. So the condition that (4.12) is obeyed for some $\epsilon$ is that

$$
\pm b_{1}^{-1} \pm b_{2}^{-1} \pm b_{3}^{-1}=0,
$$

with some set of the signs. This is in full agreement with (4.2).

\section{Acknowledgments}

This work was supported in part by NSF Grant PHY-9513835 and the Caltech Discovery Fund. I would like to thank J. Gomis for discussions. 


\section{References}

[1] J. Polchinski, Superstring theory, vol. 2, chapter 13 Cambridge University Press, Cambridge 1998.

[2] N. Seiberg and E. Witten, String theory and noncommutative geometry, J. High Energy Phys. 09 (1999) 032 hep-th/9908142.

[3] B. Chen, H. Itoyama, T. Matsuo and K. Murakami, $p p^{\prime}$ system with B field, branes at angles and noncommutative geometry, Nucl. Phys. B 576 (2000) 177 hep-th/9910263.

[4] M. Mihailescu, I.Y. Park and T.A. Tran, D-branes as solitons of an $N=1, D=10$ non-commutative gauge theory, Phys. Rev. D 64 (2001) 046006 hep-th/0011079.

[5] O. Bergman, Three-pronged strings and 1/4 BPS states in $N=4$ super-Yang-Mills theory, Nucl. Phys. B 525 (1998) 104 hep-th/9712211;

O. Bergman and B. Kol, String webs and 1/4 BPS monopoles, Nucl. Phys. B 536 (1998) 149 hep-th/9804160.

[6] A. Connes, M.R. Douglas and A. Schwarz, Noncommutative geometry and matrix theory: compactification on tori, J. High Energy Phys. 02 (1998) 003 hep-th/9711162

[7] D.J. Gross and N.A. Nekrasov, Monopoles and strings in noncommutative gauge theory, $J$. High Energy Phys. 07 (2000) 034 hep-th/0005204; Dynamics of strings in noncommutative gauge theory, J. High Energy Phys. 10 (2000) 021 hep-th/0007204.

[8] A.P. Polychronakos, Flux tube solutions in noncommutative gauge theories, Phys. Lett. B 495 (2000) 407 hep-th/0007043.

[9] D.P. Jatkar, G. Mandal and S.R. Wadia, Nielsen-Olesen vortices in noncommutative abelian Higgs model, J. High Energy Phys. 09 (2000) 018 hep-th/0007078.

[10] D. Bak, Exact solutions of multi-vortices and false vacuum bubbles in noncommutative abelian-Higgs theories, Phys. Lett. B 495 (2000) 251 hep-th/0008204.

[11] M. Aganagic, R. Gopakumar, S. Minwalla and A. Strominger, Unstable solitons in noncommutative gauge theory, J. High Energy Phys. 04 (2001) 001 hep-th/0009142.

[12] J.A. Harvey, P. Kraus and F. Larsen, Exact noncommutative solitons, J. High Energy Phys. 12 (2000) 024 hep-th/0010060.

[13] D.J. Gross and N.A. Nekrasov, Solitons in noncommutative gauge theory, J. High Energy Phys. 03 (2001) 044 hep-th/0010090.

[14] S. Kachru and J. McGreevy, Supersymmetric three-cycles and (super)symmetry breaking, Phys. Rev. D 61 (2000) 026001 hep-th/9908135].

[15] M. Berkooz, M.R. Douglas and R.G. Leigh, Branes intersecting at angles, Nucl. Phys. B 480 (1996) 265 hep-th/9606139.

[16] E.S. Fradkin and A.A. Tseytlin, Nonlinear electrodynamics from quantized strings, Phys. Lett. B 163 (1985) 123.

[17] C.G. Callan, C. Lovelace, C.R. Nappi and S.A. Yost, String loop corrections to beta functions, Nucl. Phys. B 288 (1987) 525:

A. Abouelsaood, C.G. Callan, C.R. Nappi and S.A. Yost, Open strings in background gauge fields, Nucl. Phys. B 280 (1987) 599. 
[18] D.-E. Diaconescu and R. Entin, A non-renormalization theorem for the $D=1, N=8$ vector multiplet, Phys. Rev. D 56 (1997) 8045 hep-th/9706059.

[19] O. Aharony, M. Berkooz and N. Seiberg, Light-cone description of $(2,0)$ superconformal theories in six dimensions, Adv. Theor. Math. Phys. 2 (1998) 119 hep-th/9712117.

[20] N. Nekrasov and A. Schwarz, Instantons on noncommutative $R^{4}$ and $(2,0)$ superconformal six dimensional theory, Commun. Math. Phys. 198 (1998) 689 hep-th/9802068.

[21] M. Li, Strings from IIB matrices, Nucl. Phys. B 499 (1997) 149 hep-th/9612222.

[22] H. Aoki, N. Ishibashi, S. Iso, H. Kawai, Y. Kitazawa, and T. Tada, Noncommutative Yang-Mills in IIB matrix model, Nucl. Phys. B 565 (2000) 176 hep-th/9908141.

[23] N. Ishibashi, A relation between commutative and noncommutative descriptions of D-branes, hep-th/9909176.

[24] N. Ishibashi, S. Iso, H. Kawai and Y. Kitazawa, Wilson loops in noncommutative Yang-Mills, Nucl. Phys. B 573 (2000) 573 hep-th/9910004.

[25] I. Bars and D. Minic, Non-commutative geometry on a discrete periodic lattice and gauge theory, Phys. Rev. D 62 (2000) 105018 hep-th/9910091.

[26] J. Ambjørn, Y.M. Makeenko, J. Nishimura and R.J. Szabo, Finite $N$ matrix models of noncommutative gauge theory, J. High Energy Phys. 11 (1999) 029 [hep-th/9911041; Nonperturbative dynamics of noncommutative gauge theory, Phys. Lett. B 480 (2000) 399 hep-th/0002158;

J. Ambjørn, K.N. Anagnostopoulos, W. Bietenholz, T. Hotta and J. Nishimura, Large- $N$ dynamics of dimensionally reduced $4 d \mathrm{SU}(N)$ super Yang-Mills theory, J. High Energy Phys. 07 (2000) 013 hep-th/0003208;

J. Ambjørn, Y.M. Makeenko, J. Nishimura and R.J. Szabo, Lattice gauge fields and discrete noncommutative Yang-Mills theory, J. High Energy Phys. 05 (2000) 023 hep-th/0004147.

[27] L. Alvarez-Gaumé and S.R. Wadia, Gauge theory on a quantum phase space, Phys. Lett. B 501 (2001) 319 hep-th/0006219.

[28] A.H. Fatollahi, Gauge symmetry as symmetry of matrix coordinates, Eur. Phys. J. C 17 (2000) 535 hep-th/0007023.

[29] N. Seiberg, A note on background independence in noncommutative gauge theories, matrix model and tachyon condensation, J. High Energy Phys. 09 (2000) 003 hep-th/0008013.

[30] T. Banks, W. Fischler, S.H. Shenker and L. Susskind, M-theory as a matrix model: a conjecture, Phys. Rev. D 55 (1997) 5112 hep-th/9610043.

[31] O.J. Ganor, S. Ramgoolam and I.W. Taylor, Branes, fluxes and duality in M(atrix)-theory, Nucl. Phys. B 492 (1997) 191 hep-th/9611202.

[32] T. Banks, N. Seiberg and S.H. Shenker, Branes from matrices, Nucl. Phys. B 490 (1997) 91 hep-th/9612157. 